\title{
A study of the pattern of non-venereal genital dermatoses in male patients at a Tertiary Care Centre from Hadoti region of Rajasthan, India
}

\author{
Asha Nyati, Arti Singh, Alpana Mohta, Ramesh Kumar Kushwaha, Devanshi Gupta, \\ Suresh Kumar Jain
}

Department of Dermatology, Venereology and Leprology, Government Medical College, Kota, Rajasthan, India

Corresponding author: Asha Nyati, MD, E-mail: drashanyati@gmail.com

\begin{abstract}
Background: Non venereological diseases of genitalia can be a diagnostic dilemma to a dermatologist and also a cause of considerable concern to the patient because they tend to get misdiagnosed with venereal diseases. Aims: To study the pattern and clinico-epidemiological profile of non-venereal dermatoses of male genitalia in Hadoti region of Rajasthan. Materials and Methods: We conducted this prospective, descriptive study in 250 male patients with non venereal dermatoses in Rajasthan from January 2015 to July 2016. The demographic profile and clinical findings of the patients were recorded and appropriate investigations and histopathological examination were done as and when required. Cases having venereal diseases were excluded from the study by clinical examination, serological and microbiological tests for venereal diseases. Results: The age of the patients ranged between one to 70 years, with mean age being 27.l years. The most common dermatosis was nodular scabies 54 (21.6\%) followed by sebocystoma multiplex 24 (9.6\%), fixed drug eruption 19 (7.6\%), tinea genitalis 17 (6.8\%) and genital psoriasis 14 (5.6\%) cases. Conclusion: This study highlights the importance of diagnosing non-venereal dermatoses for both correct treatment of the patient as well as to alleviate the anxiety associated with venereophobia and cancer phobia.
\end{abstract}

Key words: Non venereal genital dermatosis, Male genitalia, angiokeratoma, Sebocystoma multiplex, Pseudoepitheliomatous keratotic and micaceous balanitis, Penile horn

\section{INTRODUCTION}

Owing to the stigma attached to venereal diseases, it is quintessential to differentiate nonvenereal disorders from venereal ones, in order to avoid psychological upset and prevent disease transmission. Nonvenereal genital dermatoses (NVGD) include a multitude of dermatosis with various etiologies which are not sexually contracted.

On the basis of etiopathogenesis various studies in the past have divided non venereal dermatoses into five major groups: Inflammatory diseases, infections and infestations, congenital disorders, benign abnormalities, premalignant and malignant lesions, along with miscellaneous (unclassified) dermatoses [1]. Many a times, young men report to us with concerns over physiological variations like pearly penile papules and Fordyce spots regarding their recruitment in armed forces as they assumed it is venereal in origin. However, such patients should be explained about the benign and non-venereal nature of these diseases and counselled accordingly, and whenever necessary given medical fitness certificate.

On top of that, these diseases also tend to have varying presentations, so, a holistic approach to diagnosis and management in this field is of utmost importance. Therefore, we carried out a study to evaluate the patterns of various of nonvenereal dermatoses over genitalia in male patients in our region.

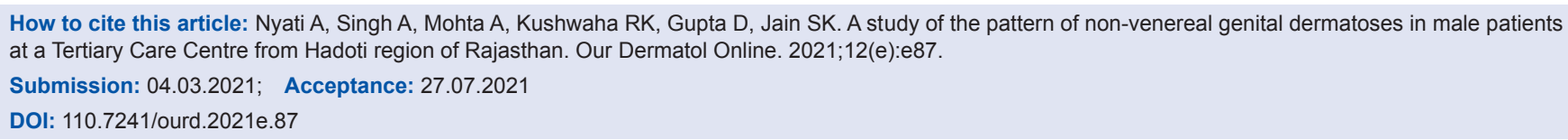




\section{MATERIALS AND METHODS}

This was a prospective, observational and descriptive study including a total of 250 male patients who presented with genital lesions of nonvenereal origin in our Tertiary Care Centre Department between the months of January 2015 to July 2016. All male patients, irrespective of their age, were examined for nonvenereal dermatoses after taking a written informed consent. A thorough history taking in the form of demographic profile, presenting complaint, duration, site and type of lesion, history of onset, history of sexual exposure and presence of any comorbidities or other cutaneous disorders was recorded on a written proforma. Patients with any venereal disease were excluded.

In case of doubt, appropriate tests from a battery of investigations like KOH mount, Gram-stain, venereal disease research laboratory test, HIV test and histopathological examination were carried out.

\section{STATISTICAL ANALYSIS}

Data was entered into SPSS version 20.0. Mean and standard deviation were calculated.

\section{RESULTS}

A total of 250 patients with non-venereal dermatoses of male genitalia were included in our study. The age of the patients ranged between zero to 70 years, with mean age being $27.1 \pm 11.3$ years. The age group involved most commonly was $0-10$ years (58 patients, $23.2 \%$ ) followed by $31-40$ years (50 patients, 20\%). While $118(47.2 \%)$ patients were unmarried, the remaining $132(52.8 \%)$ patients were married (Table 1) The total number of patients belonging to rural background were 121 (48.4\%) and urban dwellers were 129 (51.6\%) patients. The most common presenting complaint of patients was genital itching 97 (38.8\%), however 30 (12.2\%) patients were asymptomatic. Other complaints included pain, burning, redness, swelling, oozing, discharge, erosions, ulceration, and lichenification. A few patients also had a constellation of complaints. A total of 31 different types of genital dermatoses were observed by us. The most common dermatosis was nodular scabies (21.6\%) followed by sebocystoma multiplex 24 (9.6\%), fixed drug eruption 19 (7.6\%), tinea genitalis $17(6.8 \%)$ and genital psoriasis $14(5.6 \%)$. Other diseases observed were:
1) Infections and infestations: molluscum contagiosum $4(1.6 \%)$, papulonecrotic tuberculid $2(0.8 \%)$, lepromatous leprosy $2(0.8 \%)$;

2) Inflammation: lichen planus $12(4.8 \%)$, genital vitiligo $12(4.8 \%)$, irritant contact dermatitis 7 (2.8\%), lichen nitidus 5 (2\%), balanitis xerotica obliterans 4 (1.6\%), Zoon's balanitis 4 (1.6\%), Steven-Johnson syndrome/toxic epidermal necrolysis 4 (1.6\%), Reiter's disease $2(0.8 \%)$, Hailey-Hailey disease $2(0.8 \%)$, calcinosis cutis $2(0.8 \%)$, circinate balanitis $2(0.8 \%)$, fascitis $1(0.4 \%)$, pyoderma gengrenosum $1(0.4 \%)$;

3) Benign conditions/physiological variants: pearly penile papules $8(3.2 \%)$, Fordyce spots $6(2.4 \%)$, angiokeratoma $2(0.8 \%)$, dermoid cyst $1(0.4 \%)$;

4) Premalignant/malignant: bowenoid papulosis $3(1.2 \%)$, squamous cell carcinoma $2(0.8 \%)$, penile horn (with lichen sclerosus et atrophicus) $1(0.4 \%)$, and pseudoepitheliomatous keratotic and micaceous balanitis $1(0.4 \%)$;

5) Miscellaneous: dunken's dermatitis $6(2.4 \%)$ and dengue rash 1 (0.4\%), (Table 2; Table 3; Fig. 1).

\section{DISCUSSION}

The definite diagnosis of NVGD prevents unnecessary mental and conjugal tensions, associated with the taboo of venereal diseases and cancer phobia [2].

Only a few studies have comprehensively described various patterns of nonvenereal dermatoses in males. Our study is first to describe so in Hadoti area of Rajasthan. Various similar studies have been done by Saraswat et al. [1], Acharya et al. [3], to name a few.

Table 1: Martial status of patients

\begin{tabular}{lc}
\hline Marital Status & No. of Patients \\
\hline Unmarried & $118(47.2)$ \\
Married & $132(52.2 \%)$ \\
\hline
\end{tabular}

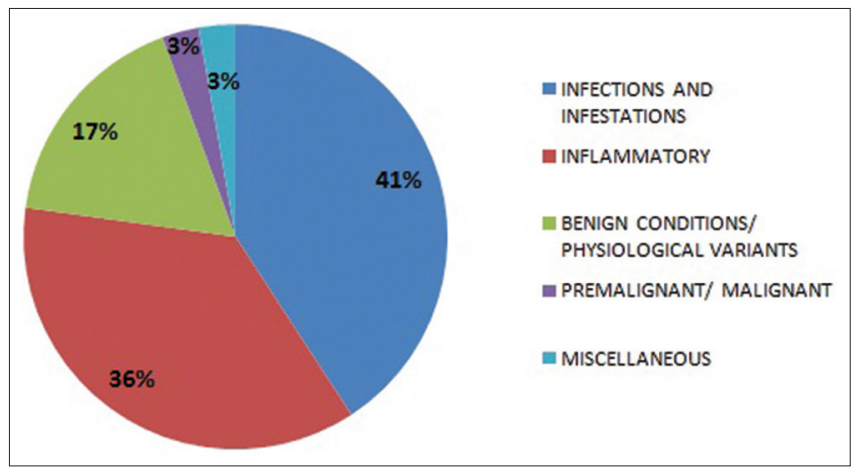

Figure 1: Etiological distribution of patients. 
Table 2: Etiological distribution of diseases

\begin{tabular}{lc}
\hline Lesions & No. (\%) \\
\hline 1. Infections And Infestations & $102(40.8 \%)$ \\
Nodular Scabies & $54(21.6 \%)$ \\
Tinea Genitalis & $17(6.8 \%)$ \\
Molluscum Contagiosum & $4(1.6 \%)$ \\
Papulonecrotic Tuberculid & $2(0.8 \%)$ \\
Lepromatous Leprosy & $2(0.8 \%)$ \\
2. Inflammatory & $91(36.4 \%)$ \\
Fixed Drug Eruption & $19(7.6 \%)$ \\
Psoriasis & $14(5.6 \%)$ \\
Lichen Planus & $12(4.8 \%)$ \\
Vitiligo & $12(4.8 \%)$ \\
Irritant Contact Dermatitis & $7(2.8 \%)$ \\
Lichen Nitidus & $5(2 \%)$ \\
Balanitis Xerotica Obliterans & $4(1.6 \%)$ \\
Zoon's Balanitis & $4(1.6 \%)$ \\
Steven-Johnson Syndrome/ Toxic Epidermal Necrolysis & $4(1.6 \%)$ \\
Hailey-Hailey Disease & $2(0.8 \%)$ \\
Reiter's Disease & $2(0.8 \%)$ \\
Calcinosis Cutis & $2(0.8 \%)$ \\
Circinate Balanitis & $2(0.8 \%)$ \\
Pyoderma Gangrenosum & $1(0.4 \%)$ \\
Fascitis & $1(0.4 \%)$ \\
3. Benign Conditions/ Physiological Variants & $43(17.2 \%)$ \\
Sebocystoma Multiplex & $24(9.6 \%)$ \\
Pearly Penile Papules & $8(3.2 \%)$ \\
Fordyce Spots & $6(2.4 \%)$ \\
Angiokeratoma & $2(0.8 \%)$ \\
Dermoid Cyst & $1(0.4 \%)$ \\
4. Premalignant/ Malignant & $7(2.8 \%)$ \\
Bowenoid Papulosis & $3(1.2 \%)$ \\
Squamous Cell Carcinoma & $2(0.8 \%)$ \\
Penile Horn (With Lichen Sclerosus Atrophicus) & $1(0.4 \%)$ \\
Pseudoepitheliomatous Keratotic And Micaceous Balanitis & $1(0.4 \%)$ \\
Discellaneous & $7(2.8 \%)$ \\
Dengue Rash & $6(2.4 \%)$ \\
\hline & $1(0.4 \%)$ \\
\hline
\end{tabular}

Table 3: site involvement

\begin{tabular}{lcccc}
\hline Sites & $\begin{array}{c}\text { Only } \\
\text { Genitalia }\end{array}$ & Orogenital & $\begin{array}{c}\text { Genital } \\
\text { and } \\
\text { Cutaneous }\end{array}$ & $\begin{array}{c}\text { Orogenital } \\
\text { and } \\
\text { Cutaneous }\end{array}$ \\
\hline $\begin{array}{l}\text { Number of } \\
\text { Patients (\%) }\end{array}$ & $128(51.2 \%)$ & $32(12.8 \%)$ & $58(23.2 \%)$ & $32(12.8 \%)$ \\
\hline
\end{tabular}

A total of 31 different nonvenereal dermatoses were observed in this study (Table 2). The overall prevalence of non-venereal genital dermatoses observed during our study period was 20.7 per 10,000 male patients attending our outpatient department which was higher than that observed by previously published work.

Most commonly affected age group was between $0-10$ years ( 58 patients-23.2\%), followed by $31-40$ years (50 patients-20\%) which was fairly comparable to the results of and Saraswat et al [1].
We divided our cases into 5 broad categories based on etiopathogenesis (Table 2). Infections and infestations accounted for the majority of $102(40.8 \%)$ patients, followed by inflammatory disorders 91 (36.4\%) and benign dermatosis 43 (17.2\%). Premalignant/malignant dermatoses accounted only for $7(2.8 \%)$ cases. Other miscellaneous conditions including dengue rash and dunken's dermatitis were seen in just 7 (2.8\%) patients.

The most common disorder observed by us was nodular scabies seen in $54(21.6 \%)$ patients, followed by genital vitiligo in 27 (10.8\%), and sebocystoma multiplex in 24 (9.6\%). (Table 2). Saraswat et al. [1] had observed genital vitiligo to be the commonest dermatosis.

Nodular scabies confined to the genital was an exclusive finding only in $21(8.4 \%)$ patients.

In our study sebocystoma multiple was seen as the second most common dermatosis with 24 (9.6\%) patients, which was slightly higher than previously published observations.

Fixed drug eruptions (FDE) were observed in 19 (7.6\%) patients. This was similar to Saraswart et al.'s [1] observation who had also reported it to the third most common dermatosis with $12 \%$ cases. In our study, the perpetrating drugs included ornidazole, tinidazole, nonsteroidal antiinflammatory drugs and ofloxacin. We also observed 4 (1.6\%) cases of Steven Johnson syndrome (l patient) and toxic epidermal necrolysis (3 patients). The culprit drugs were carbamazepine and cotrimoxazole.

In our study 17 (6.8\%) patients had tinea genitalis (12 on shaft and 5 on scrotum). Tinea genitalis is often seen in association with tinea cruris and our study was no exception[4]. Twelve out of 17 patients had concurrent tineacruris while 2 had concurrent tinea unguium of fingernails.

Genital psoriasis was seen in 14 (5.6\%) patients. Nine of those patients had lesions confined solely to the glans while the remaining 5 patients also had involvement of scrotum and other flexural sites like groin and axilla. Saraswat et al. [1] had observed genital psoriasis in 3\% cases. Up to $63 \%$ patients of psoriasis can present with genital lesions with exclusive involvement of this site in 2 to $5 \%$ cases [5]. An interesting observation in our study was the morphological variation in lesions of genital psoriasis, varying with the status of foreskin. 
Twelve patients has only slightly scaly thin plaques over shaft or glans of penis, however, the remaining two patients who were circumcised has silverly scaly plaques with mild induration.

A handful of previous reports have described an exclusive involvement of genitalia in both psoriasis and lichen planus [6]. The possible explanation for this can be the trauma induced by intercourse, fitted garments and irritant reaction by urination, all contributing to frequent koebnerization seen over this site.

Genital vitiligo accounted for $12(4.8 \%)$ cases and was seen in all age group from young adult to older age group. Lichen sclerosus of male genitalia (balanitis xerotica obliteran) was seen in $5(2 \%)$ cases ( 4 cases in inflammatory pattern and 1 in premalignant pattern with secondary cutaneous horn). It is a chronic inflammatory dermatosis associated with significant discomfort and morbidity of unknown etiology. Saraswat et al. [1] had reported similar results with 3 cases. Two of our patients had phimosis and were advised circumcision, one had urinary hesitancy and burning.

Amongst rest of the infective etiologies, 4 (1.6\%) children presented to us with molluscum contagiosum of shaft of penis and scrotum. All of them also had similar lesions over other body parts like face or trunk. Molluscum contagiousm is one of the most common diseases associated with pseudo-koebnerization [7] and after ruling out any signs of sexual abuse all 4 of these causes were diagnosed to be a result of autoinnoculation.

Two cases of Papulonecrotic tuberculid also presented to us with multiple punched out non healing painless ulcers over the scrotum. After confirming the diagnosis through Mantoux test positivity and histopathological examination the patients were put on anti tubercular drugs (Fig. 2).

We also observed 3 cases of lepromatous leprosy with genital lesions. In most cases, lesions are reported to be present on scrotum with or without penile involvement.

Pearly Penile Papules were present in 8 (3.2\%) of our patients. These lesions are seen over the coronal sulcus as multiple flesh colored to slightly hypopigmented papules. Most of the patients were under 30 years of age (6 out of 8 ) and were concerned about the origin of the disease with 2 assuming it to be sexual in origin.
We also observed 6 cases of Fordyce spots (Fig. 3). While pearly penile papules are confined only to the head of the penis, Fordyce spots involve the shaft or scrotum $[8,9]$. All these patients were explained about the benign and non-venereal nature of these diseases and counselled accordingly.

Zoon's balanitis or plasma cell balanitis was observed in $2(0.8 \%)$ cases (Fig. 4$)$. While Saraswat et al. [1] had reported $2 \%$ of such cases. It is a disorder of old age in uncircumcised male with an unknown etiology [10].

Terra firma-forme dermatosis, also known as Dunken's dermatitis, is characterized by brown-grey cutaneous patches or plaques which can simply be eradicated by forceful swabbing with alcohol pads. The pathogenesis involves abnormal and delayed keratinization. The sites most commonly involved are neck, ankles, and face, however, rarely it can also involve genitalia [11]. We

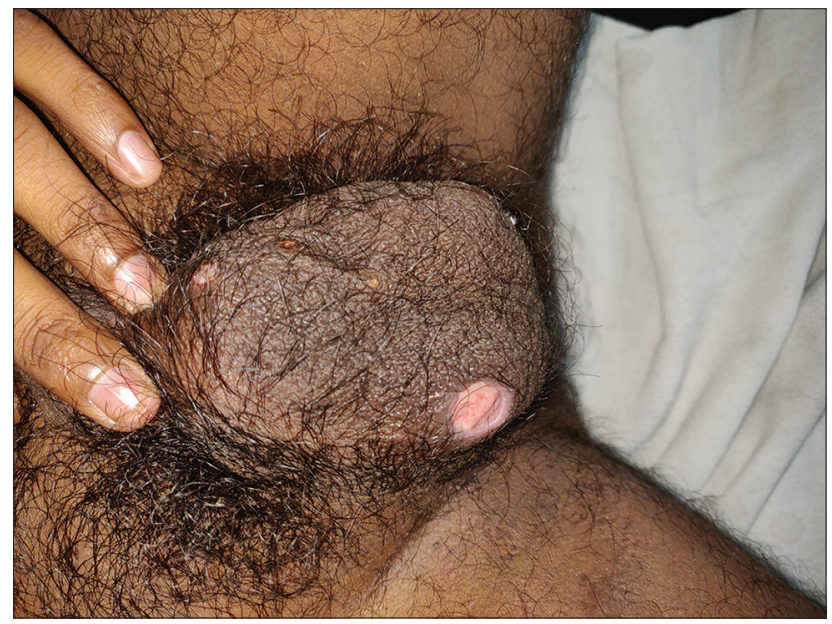

Figure 2: Papulonecrotic tuberculid.

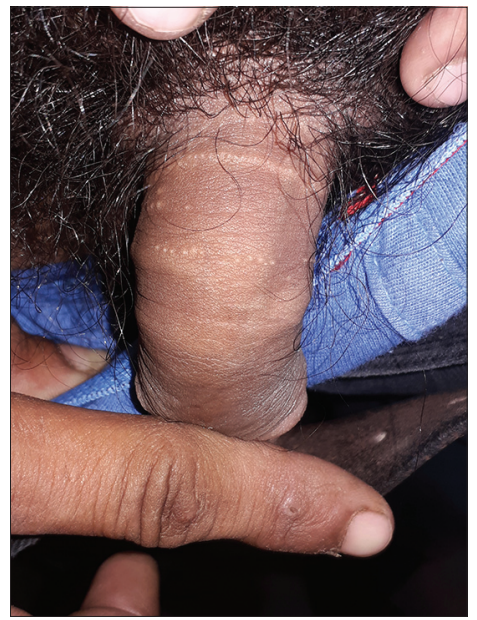

Figure 3: Fordyce spots. 


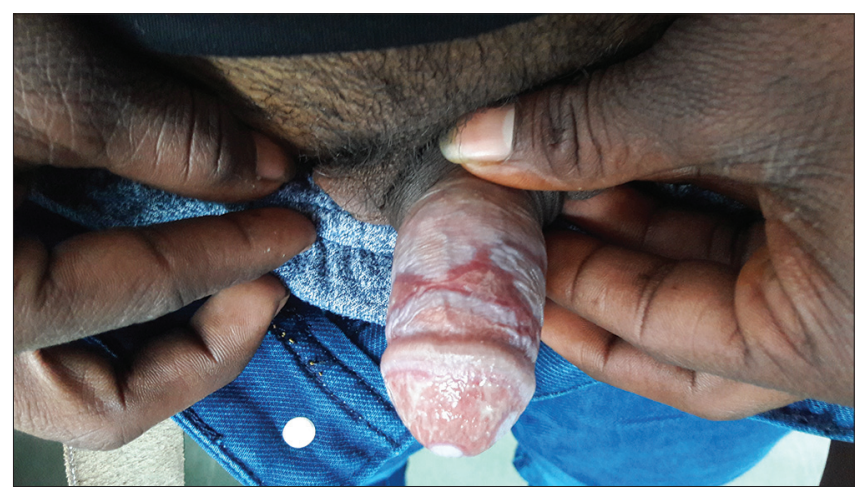

Figure.4: Zoon's balanitis.

encountered $6(2.4 \%)$ such cases. Four out of 6 cases were under 10 years of age.

Genital angiokeratomas are benign, idiopathic, vascular lesions. Angiokeratomas of Fordyce are seen most commonly over the scrotum and glans penis at times [12]. We encountered $2(0.8 \%)$ such cases.

We also found $1(0.4 \%)$ case of Dengue rash on scrotum. Our patient presented with non pruiritic lesions over scrotum with intense itching in palms and soles. It can lead to scrotal and penile edema in dengue.

We observed $1(0.4 \%)$ case of genital pyoderma gangrenosum. Pyoderma gangrenosum (PG) is a rare, chronic, often destructive, inflammatory skin disease characterized by painful nodule or pustule which breaks down to form a progressively enlarging ulcer with a raised, tender, undermined border which can very rarely also involve genitals [13].

One case $(0.4 \%)$ of Pseudoepitheliomatous keratotic and micaceous balanitis (PKMB) also presented to us (Fig. 5). PKMB is an extremely rare, clinical entity characterized by mica-like crusts and keratotic horny masses on glans penis with an unknown etiology [14].

Two of the patients also presented with calcinosis cutis of scrotum. One of them had developed calcinosis over pre existing lesions of sebaceous cysts. Surgical excision was advised in both cases.

Circinate balanitis presents as a well-demarcated, moist, erythematous plaque with a ragged or scalloped white border on the glans penis [15]. Two such cases presented to us (aged 26 and 31 years) one was associated with Reiter's disease while the other was idiopathic in origin (which responded with oral ciprofloxacin).

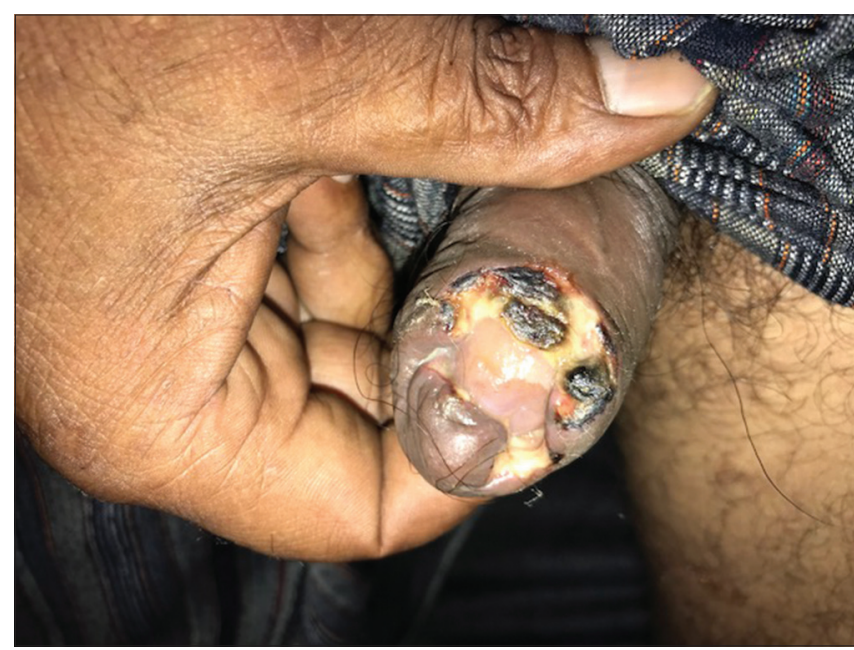

Figure 5: Pseudoepitheliomatous keratotic and micaceous balanitis.

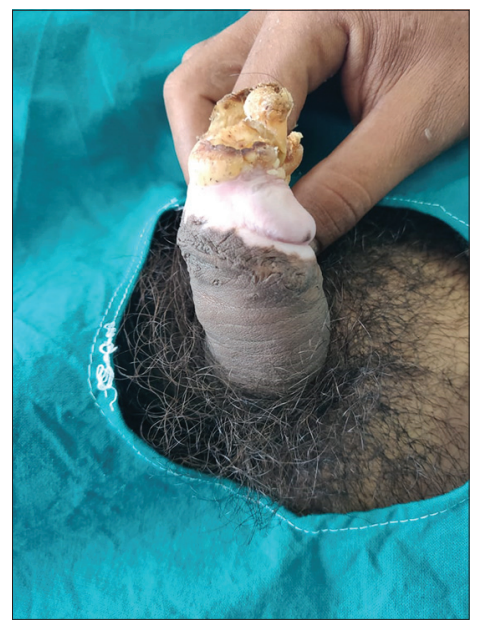

Figure 6: Cutaneous horn on lichen sclerosus et atrophicus.

Amongst premalignant and malignant dermatoses, 4 cases each of bowenoid papulosis and 3 of squamous cell carcinoma were identified after histopathological confirmation.

One $(0.4 \%)$ case of lichen sclerosus et atrophicans with superimposed penile horn was also observed (Fig. 6). Cutaneous horn has been described at various sites, but its occurrence on penis is very rare and unusual. It has been attributed to chronic irritation, phimosis, and trauma and can be premalignant in nature. Other rarely observed diseases have been tabulated in Table 2 .

\section{CONCLUSION}

It is not surprising that genitalia have a higher tendency to present with atypical morphologies of common dermatosis owing to the male external genitalia's characteristic anatomy which is prone to friction, 
heat and maceration. A prompt arrival of diagnosis for non-venereal dermatosis can only be done after being thoroughly aware of the prevalence, clinical and etiological factors responsible for it. A physician must always adopt an impartial approach while dealing with any patient presenting with genital lesions. The taboo of venereophobia can be uplifted only if the patients stop feeling ashamed while seeking a health practitioner for such diseases. While explaining the patient about the benign nature of the disease can help him in leading a better social life, in case of premalignant and malignant dermatosis a rapid and definite diagnosis can avoid unnecessary morbidity and mortality. Although our study was a helpful tool for understanding the pattern of non venereal male genital dermatoses in our region, the absence of any congenital disease and a short study period were the limitations encountered by us.

\section{Statement of Human and Animal Rights}

All the procedures followed were in accordance with the ethical standards of the responsible committee on human experimentation (institutional and national) and with the 2008 revision of the Declaration of Helsinki of 1975.

\section{Statement of Informed Consent}

Informed consent for participation in this study was obtained from all patients.

\section{REFERENCES}

1. Saraswat PK, Garg A, Mishra D, Garg S. A study of pattern of nonvenereal genital dermatoses of male attending skin OPD at a tertiary care center. Indian J Sex Transm Dis AIDS. 2014;35:129-34.

2. Nyati A, Agarwal P. Pattern of non-venereal dermatoses of female external genitalia in Rajasthan. Asian Pac J Health Sci. 2016;3:249-65.

3. Kumar PS, Ramatulasi S, Darla S, Acharya A, A clinical study on non venereal genital dermatoses in adult males at a tertiary care center. Indian J Clin Exp Dermatol. 2019;5:98-102.

4. Luchsinger I, Bosshard PP, Kasper RS, Reinhardt D, Lautenschlager S. Tinea genitalis: a new entity of sexually transmitted infection? Case series and review of the literature. Sex Transm Infect. 2015;91:493-6.

5. Beck KM, Yang EJ, Sanchez IM, Liao W. Treatment of genital psoriasis: a systematic review. Dermatol Ther (Heidelb). 2018;8:509-25.

6. Zendell K. Genital lichen planus: update on diagnosis and treatment. Semin Cutan Med Surg. 2015;34:182-6.

7. Elboukhari K, Achehboune K, Elloudi S, Baybay H, Mernissi FZ. A strange presentation of molluscum contagiosum in an immunocompetent child. Our Dermatol Online. 2020;11:212-3.

8. Dobrev HP, Nocheva DG. Videodermatoscopy of pearly penile papules. Case reports. Our Dermatol Online. 2015;6:29-31.

9. Lee JH, Lee JH, Kwon NH. Clinicopathologic Manifestations of Patients with Fordyce's Spots. Ann Dermatol. 2012;24:103-6.

10. Bari O, Cohen PR. Successful management of Zoon's balanitis with topical mupirocin ointment: a case report and literature review of mupirocin-responsive balanitis circumscripta plasmacelluaris [published correction appears in Dermatol Ther (Heidelb). 2017 Jun;7(2):211]. Dermatol Ther (Heidelb). 2017;7:203-10.

11. Khalidi M, Machan A, Hjira N, Boui M. Terra firma-forme dermatosis: Case report and review. Our Dermatol Online. 2021;12(e):e52.

12. Sharquie K, Jabbar RI. Classification and clinical evaluation of the types of angiokeratoma. Our Dermatol Online. 2021;12:130-4.

13. Philip M, Samson JF, Simi PS, Nandakumar G, Mathew B. Penile pyoderma gangrenosum. Indian J Sex Transm Dis AIDS. 2013;34:138-40.

14. Hanumaiah B, Mohan, Lingaiah NB, Kumaraswamy SK, Vijaya B. Pseudoepitheliomatous keratotic and micaceous balanitis: A rare condition successfully treated with topical 5-fluorouracil. Indian J Dermatol 2013;58:492.

15. Kumar S, Mahajan BB, Ahluwalia RS, Boparai AS. Reiter's disease: Circinate balanitis as alone preceding presentation - Successfully treated with pimecrolimus 1\% cream. Indian J Sex Transm Dis AIDS. 2015;36:70-3.

Copyright by Asha Nyati, et al. This is an open access article distributed under the terms of the Creative Commons Attribution License, which permits unrestricted use, distribution, and reproduction in any medium, provided the original author and source are credited.

Source of Support: Nil, Conflict of Interest: None declared. 